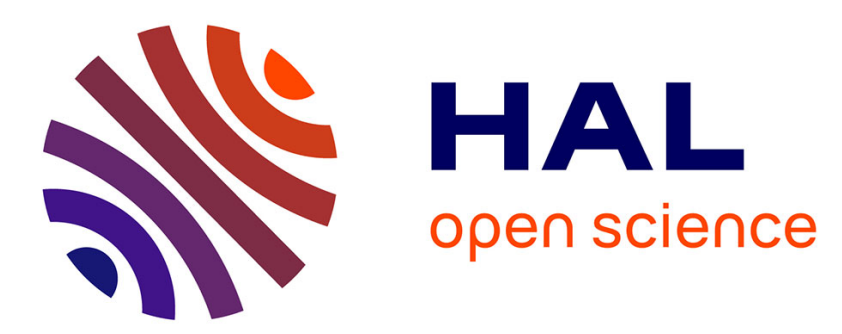

\title{
Detection and Spatial Analysis of Hepatic Steatosis in Histopathology Images using Sparse Linear Models \\ Nazre Batool
}

\section{To cite this version:}

Nazre Batool. Detection and Spatial Analysis of Hepatic Steatosis in Histopathology Images using Sparse Linear Models. IPTA 2016 - Sixth International Conference on Image Processing Theory, Tools and Applications, Dec 2016, Oulu, Finland. hal-01419385

\section{HAL Id: hal-01419385 \\ https://hal.inria.fr/hal-01419385}

Submitted on 19 Dec 2016

HAL is a multi-disciplinary open access archive for the deposit and dissemination of scientific research documents, whether they are published or not. The documents may come from teaching and research institutions in France or abroad, or from public or private research centers.
L'archive ouverte pluridisciplinaire HAL, est destinée au dépôt et à la diffusion de documents scientifiques de niveau recherche, publiés ou non, émanant des établissements d'enseignement et de recherche français ou étrangers, des laboratoires publics ou privés. 


\title{
Detection and Spatial Analysis of Hepatic Steatosis in Histopathology Images using Sparse Linear Models
}

\author{
Nazre Batool \\ School of Technology and Health, KTH Royal Institute of Technology, Stockholm, Sweden \\ e-mail: nazre.batool@sth.kth.se
}

\begin{abstract}
Hepatic steatosis is a defining feature of nonalcoholic fatty liver disease, emerging with the increasing incidence of obesity and metabolic syndrome. The research in image-based analysis of hepatic steatosis mostly focuses on the quantification of fat in biopsy images. This work furthers the image-based analysis of hepatic steatosis by exploring the spatial characteristics of fat globules in whole slide biopsy images after performing fat detection. An algorithm based on morphological filtering and sparse linear models is presented for fat detection. Then the spatial properties of detected fat globules in relation to the hepatic anatomical structures of central veins and portal tracts are explored. The test dataset consists of 38 high resolution images from 21 patients. The experimental results provide an insight into the size distributions of fat globules and their location with respect to the anatomical structures.
\end{abstract}

Keywords-Hepatic steatosis, nonalcoholic fatty liver disease, liver fat detection, spatial analysis, digital pathology, biopsy image analysis, sparse linear models, shape classification, dictionary-based algorithm.

\section{INTRODUCTION}

Nonalcoholic fatty liver disease (NAFLD) is emerging as one of the most common causes of chronic liver disease, paralleling the rise in the incidence of obesity, diabetes and the metabolic syndrome. The defining histological abnormality of NAFLD is hepatic steatosis, which refers to excessive fat accumulation in hepatocytes as fat globules. The traditional investigation of NAFLD by a pathologist involves tedious eye examination of liver biopsy slides through microscope which is significantly subjective (as was the case for our dataset as well) and time-consuming. This has motivated the imagebased evaluation of liver steatosis in the last decade after the emergence of digital microscopic scanners and digital biopsy images.

The image analysis of microscopic liver biopsy slides has mostly focused on the detection and quantification of fat globules [2], [3], [4], [5], [6], [7], [9]. There has been minimal work on the analysis of spatial and geometric characteristics of hepatic fat globules such as globule sizes, spatial distribution, clustering/spreading in relation to other anatomical tissue structures, or relation to other modalities (e.g MRI images, histological stains other than H\&E). In earlier work ( [6], [7]), relatively simpler algorithms of image morphometry were used to quantify steatosis using the area measurements of fibrosis, steatosis, parenchyma, and other anatomical structures provided by commercial software. Later work involved more advanced image processing and machine learning algorithms. For example, Liquori et al. [4] used an algorithm based on morphological properties and color segmentation. Ishikawa et al. [3] proposed to use a total of 19 image features (10 shape features, 6 brightness features and 3 tissue features) to extract fat droplets and quantify steatosis regions. Nativ et al. [5] conducted the detection of large fat droplets (macrovesicular steatosis) based on Active Contour Models and a 'nucleus adjacency' parameter introduced to quantify the displacement of cell nuclei by fat droplets. Kong et al. [10] proposed a system to quantify steatosis areas in pediatric liver biopsy images and reported correlation analysis between automatic fat detection algorithm and MRI analysis for the same dataset. A semi-supervised algorithm was proposed in [8] where user annotations were used to separate image regions of interest (i.e. having steatosis) in whole-slide biopsy images. These regions were processed using morphological filters, signed distance transform and watershed algorithm for detection of fat globules. On a different note, recently, Deng et al. [2] questioned the usual practice of comparing image analysis results with those of a pathologist's analysis as the pathologist's scores are not always objective and reproducible. In terms of spatial analysis, it is known that the hepatic fat lesions can have non-uniform spatial distribution in severe cases [1] which can introduce sampling error in fat quantification in clinical examinations resulting in mis-diagnosis. Two studies ( [12], [13]) analyzed pairs of hepatic biopsies for variation in fat quantification scores. In a more recent work, MRI images of liver were used for more detailed spatial analysis of

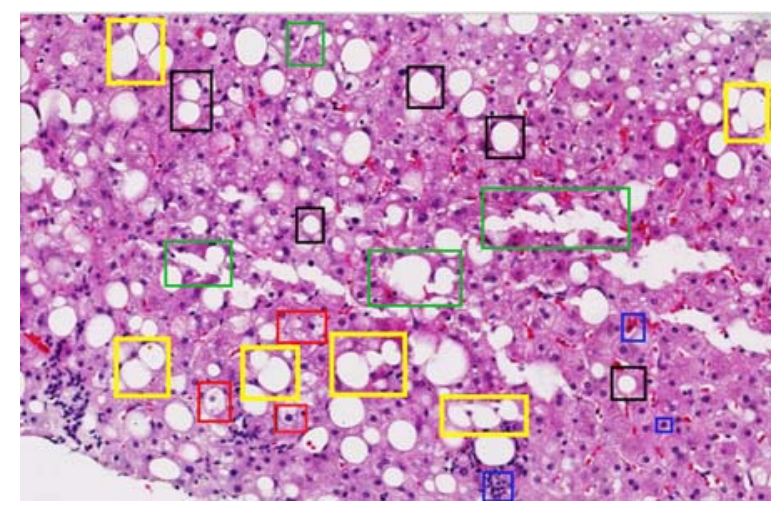

Fig. 1. Different shapes of fat globules and other typical features present in a biopsy image. (Red) Pathological cells with 'ballooning'. (Green) Damaged tissue. (Black) Fat globules detected via morphological filtering. (Yellow) Merging/clustering/elliptical fat globules NOT detected via morphological filtering. (Blue) Other tissue structures such as nuclei, red blood cells. 


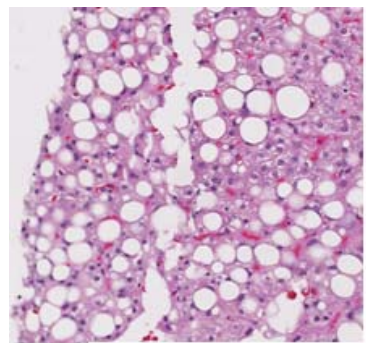

(a)

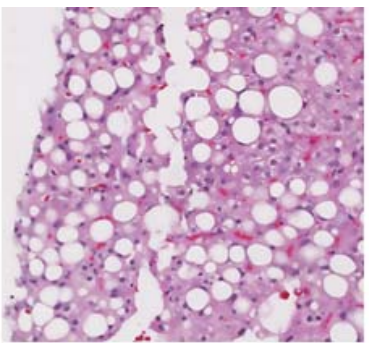

(b)

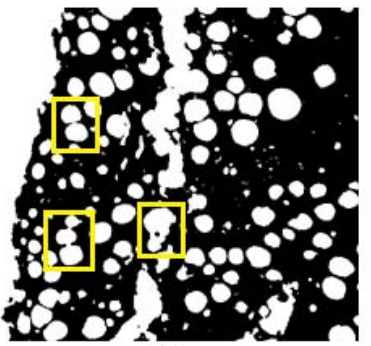

(c)

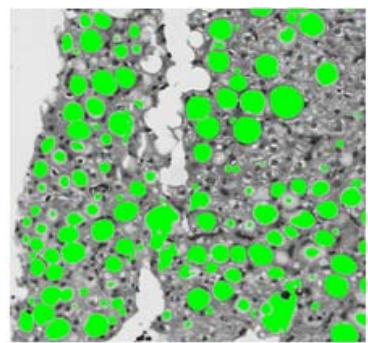

(d)

Fig. 2. Detection of fat globules. (a) Original image. (b) After bilateral filtering. (c) After thresholding of green channel (Non-circular binary shapes are enclosed in yellow). (d) Detected globules are shown in green in the green channel.

fat distribution variability among different liver lobules [14]. The interested reader is referred to [17] for a recent and comprehensive review of both clinical and automated image analysis based approaches to quantify and evaluate fatty liver diseases using different medical imaging modalities.

In this work we further the analysis of liver steatotis beyond detection and fat quantification. We propose to use a novel dictionary based algorithm for binary shape classification to improve fat globule detection results in the challenging image scenarios. The main contribution is in terms of analysis of (1) globule sizes and (2) spatial distribution of fat globules with respect to the anatomical structures in whole slide biopsy images. We hope that this preliminary analysis will pave the way for further investigation into the spatial characteristics of liver steatosis for the possible presence of any biomarkers.

\section{Methodology}

For spatial analysis, the locations of fat globules and anatomical structures i.e. hepatic vein and portal tract (CV and PT) within biopsy images are required. Liver CV's and HT's are $3 \mathrm{D}$ anatomical structures and can appear very differently in biopsies depending on the biopsy location within liver. For this

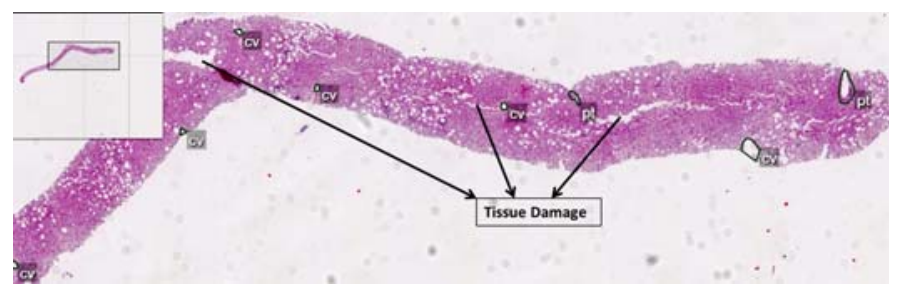

Fig. 3. Pathologist's hand-drawn anatomical markup (CV's and PT's). Some tissue damage during biopsy preparation is also highlighted. An overview of complete biopsy image can be seen in the top-left corner.

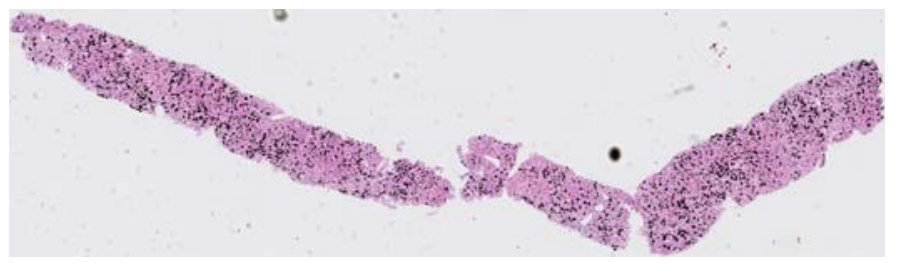

Fig. 4. Detected fat globules are shown in black in a whole biopsy image.

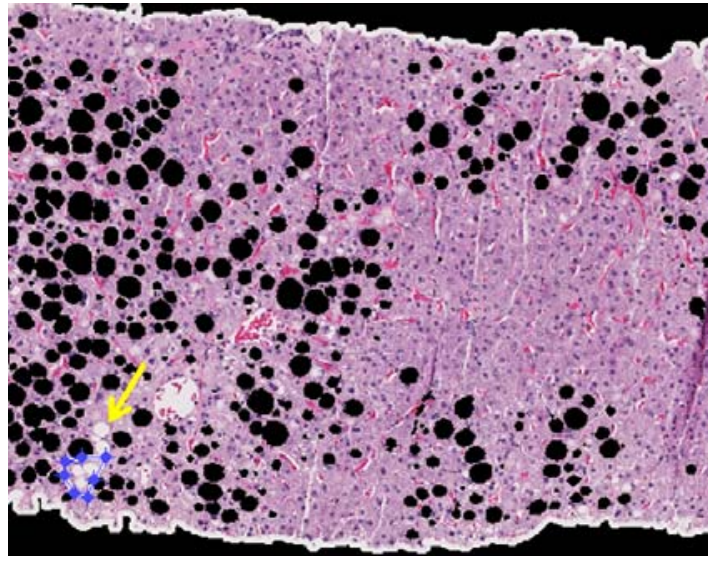

Fig. 5. Detected fat globules are shown in black for a biopsy image patch where the yellow line indicates the user-drawn polygon to highlight the missed cluster of globules.

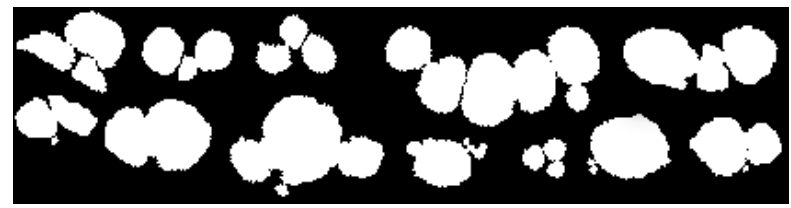

Fig. 6. Some examples of the binary shapes representing clustered fat globules in dictionary.

work, the anatomical markup was provided by a pathologist using a proprietary software used for annotating biopsies. Fig. 3 shows hand-drawn annotations for liver anatomical structures in one biopsy. The number of fat globules within a liver biopsy can vary from none to several hundreds depending on the degree of steatosis. Manual delineation of the boundaries of such a large number of globules is tedious for any pathologist. Hence, we rely on the automatic detection to delineate boundaries of fat globules. Typically, liver fat appears as oval, white globules/blobs (marked in black boxes in Fig. 1) in the surrounding pinkish tissue with purple nuclei, reddish blood cells and can be assumed easy to detect. However, the detection becomes challenging in the presence of tissue damage, pathological cells exhibiting a condition called 'ballooning' or fat globules which are adjacent/connected, have large sizes or exhibit more elliptic shapes. Fig. 1 includes 


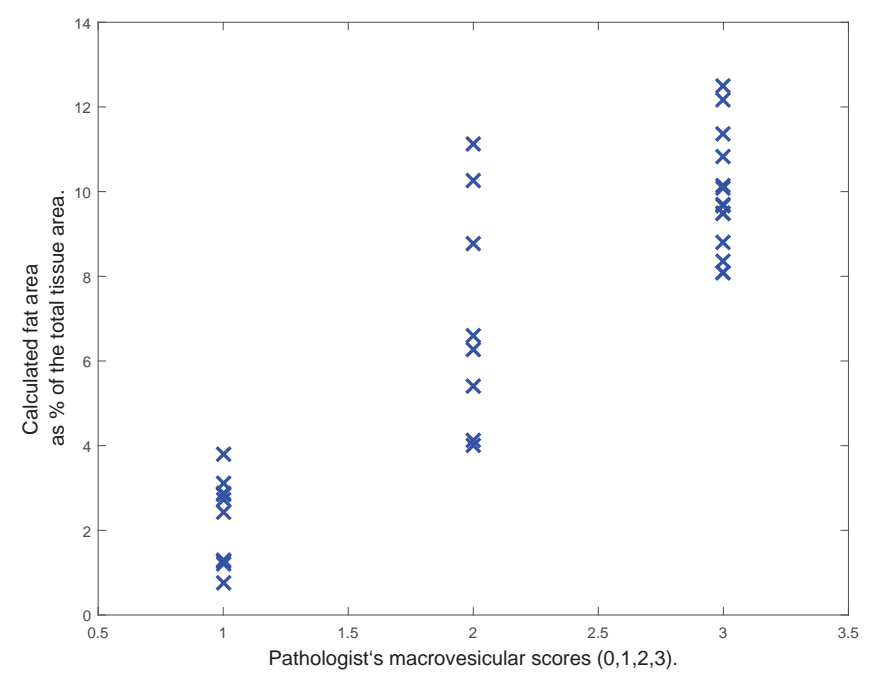

Fig. 7. Plot between pathologist's macrovesicular steatosis scores (0-3) vs. percentage scores calculated using our 2-step algorithm.

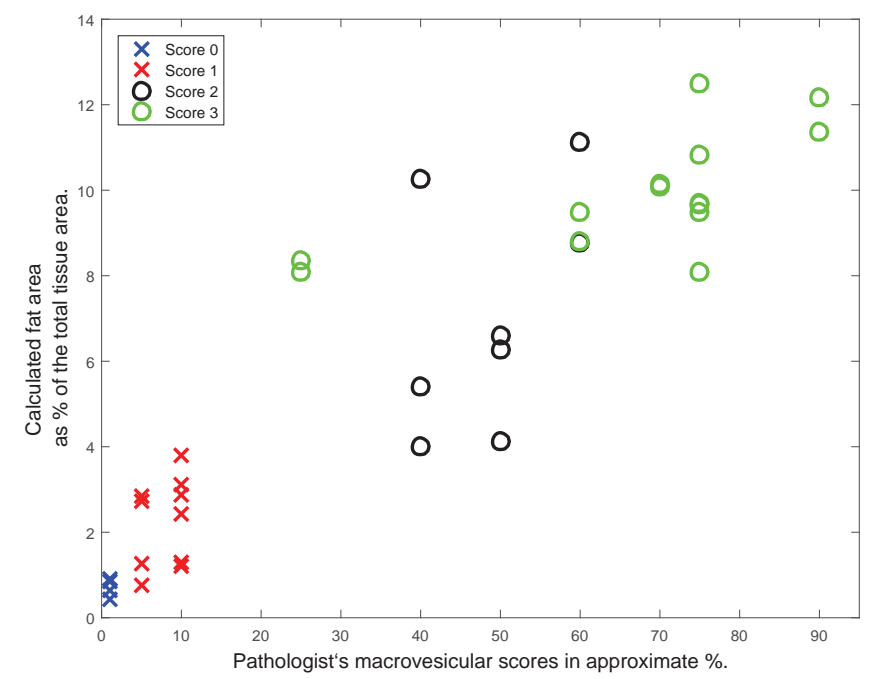

Fig. 8. Plot between pathologist's macrovesicular scores in approximate percentage vs. percentage scores calculated using our 2-step algorithm.

examples of such challenging features for a biopsy image.

To the best of our knowledge, there is no liver steatosis detection algorithm available publicly or shared for research. Hence, we conducted steatosis detection experiments using previously suggested methodologies such as morphological processing and active contour models specifically in the absence of any training dataset. But these methods did not produce accurate results in the presence of tissue damage/ballooning and clustering globules. Hence we propose to use a dictionary based algorithm for steatosis detection in this work. The advantages of such a method are twofold. First, we can include the binary shapes of interest of any type to the dictionary. And second, the detection can be carried out in a semi-supervised, online learning manner, where we need not know all the possible globule shapes a priori.

\section{A. 2-step Fat Detection Algorithm}

We propose a 2-step detection algorithm based on image morphology and sparse shape recovery using binary shape dictionary and sparse linear models. As preprocessing steps, bilateral filtering [15] is used for edge preserving smoothing to smooth image intensity in the interiors of fat globules. The typical reddish/violet hues of tissue in biopsy images with H\&E stain can be separated best from white image regions in the green channel. Hence, the thresholding of the bilateral-filtered image is done in green channel, with threshold $t_{g} \in[0,255]$, to retrieve white image regions. The resulting binary image, say $\mathbb{I}_{b}$, contains erroneous white regions due to tissue damage, anatomical tracts, pathological cell ballooning and empty slide background as well in addition to fat globules. Fig. 2 depicts different pre-processing stages for an example image. After pre-processing, our 2-step fat detection algorithm aims at filtering image regions in $\mathbb{I}_{b}$ with specific binary shapes representing fat globules. As the first step of the algorithm, simple morphological filtering based on the geometric features of 'solidity', 'eccentricity' and 'size' of the connected components is applied on $\mathbb{I}_{b}$. The high solidity and low eccentricity filter circular shapes whereas the size is selected to reject image regions which are too small to be significant or too big to be fat globules. These bigger regions are more probable to represent liver anatomical structures or tissue damage. During this step, typical false positives occur due to the binary shapes which, despite appearing circular, do not correspond to the fat globules. The typical false negatives (or missed true instances) occur due to the merging, clustering or elliptical fat globules as were highlighted in Fig. 1. Next, as a second step, we propose to use a dictionary-based binary shape recognition algorithm to reduce false negatives (or missed positive instances).

In the second step, a dictionary of binary shapes representing typically missed globules is utilized. The fat detection is formulated as a non-parametric binary shape classification problem where a test shape is represented as a sparse linear combination of dictionary items [11]. Since the binary shapes of globules can be represented as small images, the scalar fields of signed distance functions (SDF) were deemed sufficient as lossless and explicit shape features. Let a binary shape be represented as a mapping from 2D image space to a binary value, $A: \mathbb{R}^{d_{1}} \times \mathbb{R}^{d_{2}} \rightarrow\{0,1\}$, i.e. the binary value is set to one to represent the shape (See Fig. 6). The binary shape is assumed to be aligned in the middle of the image. Next, the SDF, $D_{d_{1} \times d_{2}}(A)$, associated with the binary shape is calculated and stacked to make a large vector $\left\{\phi \in \mathbb{R}^{N} ; N=d_{1} \times d_{2}\right\}$. The dictionary of $K$ binary shapes is represented as a matrix:

$$
\boldsymbol{\Phi}_{N \times K}=\left[\phi_{1}, \phi_{2}, \phi_{3}, \cdots, \phi_{K}\right] .
$$

Let $y \in \mathbb{R}^{N}$ be a test binary shape represented as the vectorized SDF, and $x \in \mathbb{R}^{K}$ be a sparse vector indicating the contribution of each atom in the dictionary while representing the test shape i.e. $y=\boldsymbol{\Phi} x$. Assuming the space of elements is closed with respect to linear combinations, $x$ is assumed to be 
of sparsity $s$ i.e. $\left(\|x\|_{0}=s\right)$. Then the sparse shape recovery can be written as the optimization problem as follows:

$$
\hat{x}=\arg \min _{x}\|x\|_{0} \text { subject to } y=\boldsymbol{\Phi} x .
$$

For the estimation of $x$, the above optimization problem with linear constraints is changed to the convex optimization problem as follows:

$$
\hat{x}=\arg \min _{x}\|\phi x-y\|_{2} \text { s.t. }\|x\|_{1}=1 \text { and } x \geq 0,
$$

and $\hat{x}$ are estimated using a quadratic programming method. The details of the estimating algorithm can be found in [11]. Commonly, this sparse shape recovery formulation is used to assign a class to the given sample by selecting the class whose dictionary atoms minimize the residual between the test shape and approximate shape using those atoms. However, in our case we assume all the dictionary atoms to belong to one class (i.e. binary shapes representing merging/elliptical fat globules) and we need to decide if a given test shape can be represented sufficiently in terms of the the dictionary atoms or not. A test shape is considered to be included in the dictionary if $\sum_{i=1}^{K} x_{i}>t_{c}$ for some threshold $t_{c}$. Since histopathological images are huge and not all the fat globules can be annotated manually to be included to the dictionary, the task of detecting binary exemplar shapes to be added to the dictionary is carried out in a semi-supervised way similar to 'online training' in machine learning algorithms. Instead of trying to determine and include all the possible missed globule instances a priori to the dictionary, a user goes over the detection results for each slide and manually annotates the image portions consisting of the missed globules as can be seen as yellow annotations in Fig. 5. The annotated image is then thresholded, aligned and represented as the vectorized SDF to be included to the dictionary. For shape alignment, the centroid of a given shape is translated to the image center and its major axis is aligned with the $\mathrm{x}$-axis. This way, as the number of dictionary items increases with the processing of more test images, the rate of false negatives decreases. Fig. 6 shows some examples of binary shapes included in the dictionary.

\section{EXPERIMENTS AND RESULTS}

Our dataset consisted of 38 needle liver biopsy images from 21 patients (dataset gathered under the Swedish ethical permit [M72-07 T5-08]). The biopsy slides included a single needle biopsy or a pair of needle biopsies. Two images were generated for each slide with two biopsies. For each slide, scoring data including macrovesicular fat severity scores (0-3) from one pathologist and one hepatologist and approximate fat area percentage scores given by a pathologist were also available. The original biopsy images of size around 100,000 x 210,000 pixels were resized with a factor of 0.2 . The resizing factor was heuristically chosen for computational efficiency while preserving the image analysis results. Detection of fat globules was performed in image patches while spatial analysis was

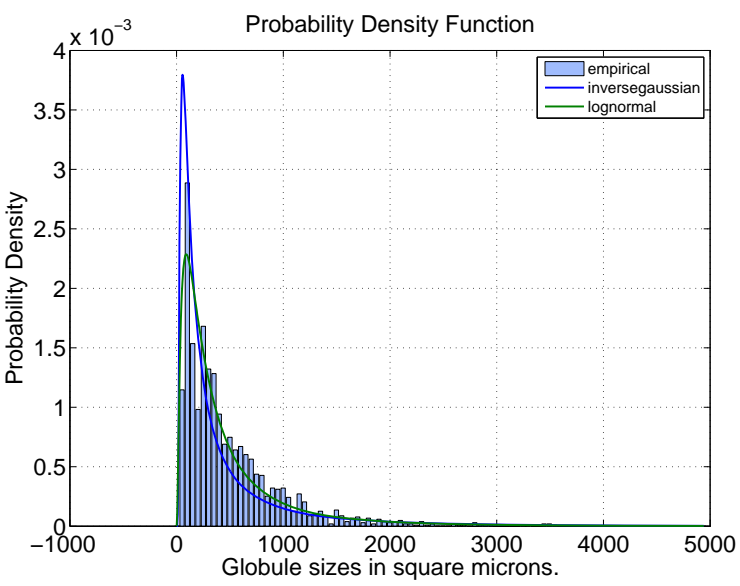

Fig. 9. A typical histogram of fat globule sizes with fitted distributions.

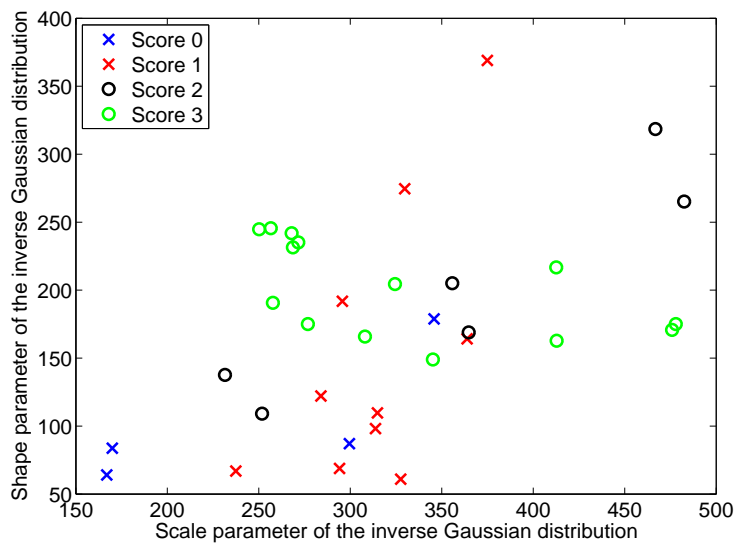

Fig. 10. Parameters (location, scale) for the fitted inverse Gaussian distributions.

performed on the whole biopsy images. For the first step of the algorithm, the threshold of green channel was selected as 200 , the morphological feature of eccentricity had to be less than 0.85 and that of solidity had to be greater than 0.85 . The connected components of the area of less than 30 pixels or more than 2700 pixels were rejected where one image pixel is equivalent to 1.15 micron and the area of one pixel square is equal to 1.3225 square microns in the biopsy slide. For the second step, dictionary atoms were fixed to be of size $255 \times 255, t_{c}$ was 0.98 and the sparsity parameter $s$ was set to be 15 .

\section{A. Validation via Correlation with Other Scoring Methods}

For validation of detection results, different approaches have been adopted previously e.g. correlation with lipid \% acquired from MRI images [4], biochemical analysis, pathologist's 4 level scores [2], and pathologist's macrovesicular \% area scores [5]. However, visual scoring by a pathologist is subjective and for our dataset the two sets of scores provided by one hepatologist and one pathologist did not match for 9 out of 38 samples. Interestingly, for 8 out of 9 disagreed samples, 

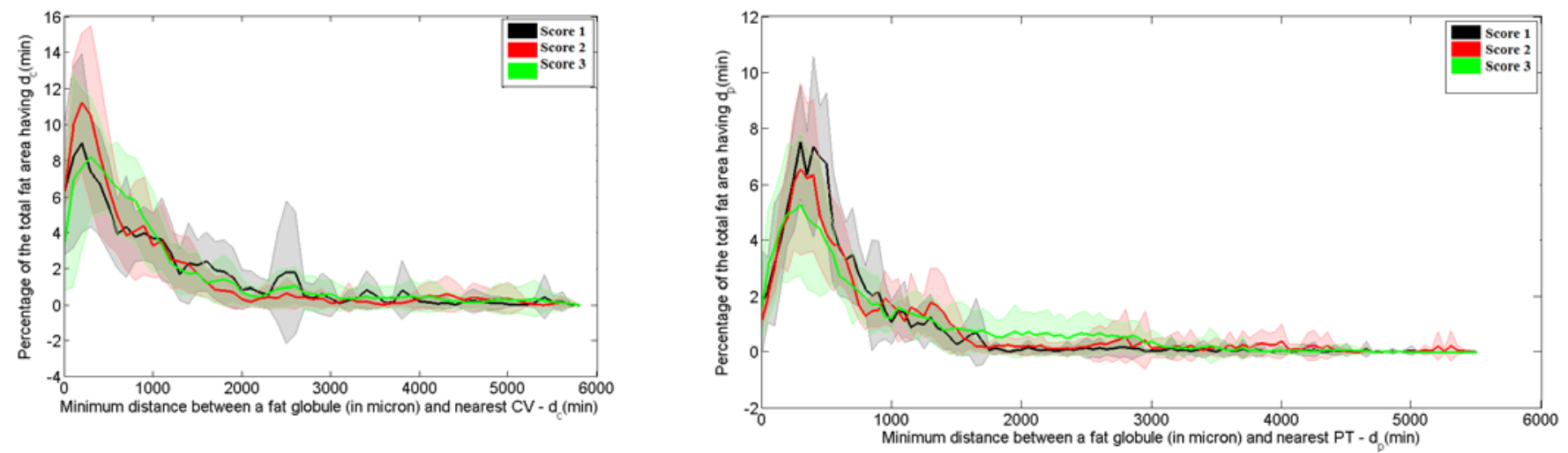

Fig. 11. Plot between the distance to the nearest PT/CV and the percentage of total fat volume lying at the distance. (Left) For PT (Right) For CV - For each score, solid line represents the average and the top and bottom boundaries of shaded region represent maximum and minimum values respectively.

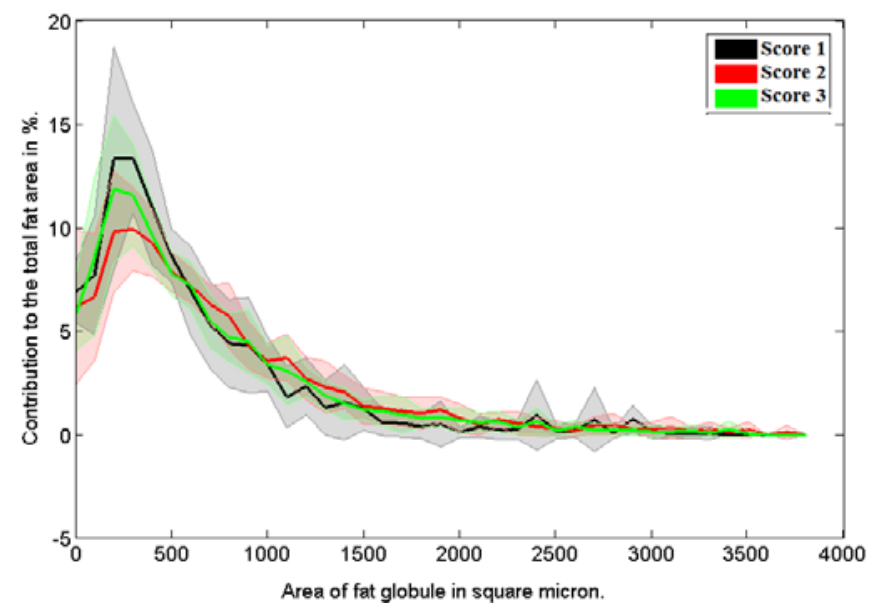

Fig. 12. Plot of globule size vs. total fat area contributed by the globules of that size (in percentage)- For each score, solid line represents the average and the top and bottom boundaries of shaded region represent maximum and minimum respectively.

the hepatologist's scores were one level lower than those given by the pathologist. Nevertheless, we selected the pathologist's 4-point and approximate area percentage scores for validation. Out of 38 images, the the number of images having scores of $0,1,2$ and 3 were $4,10,10$ and 14 respectively. Few slides with score 0 were present in the dataset because the biopsy slides having no fat globules were not very relevant to the aim of this work. Fig. 7 includes a plot between the pathologists scores and the area \% scores given by the detection algorithm. As can be observed, the algorithm \% scores show good correlation with the pathologist's scores. Statistically, the Pearson's correlation coefficient between the algorithm's scores and the pathologist's scores was 0.9066 . In terms of fat area percentages, for majority of images, the score 0 corresponded to $<1 \%$, score 1 to $1-3 \%$, score 2 to $3-8 \%$ and score 3 to $>8 \%$. Fig. 8 includes a plot between the area $\%$ scores of the algorithm and the approximate $\%$ scores given by the pathologist. The Pearson's correlation coefficient between the two percentage scores was 0.774 . After validation, we conducted further experiments to analyse the spatial characteristics of fat globules as described next.

\section{B. Sizes (Volumes) of Fat Globules}

In this set of experiments, we investigated two properties of fat globules: (a) if the globule sizes, irrespective of their locations, can be approximated by a modular probability distribution and (b) the proportion of fat area contributed to the total fat area by the globules of different sizes. The typical range of fat globule sizes was found to be 5-2000 square microns. Based on the globule size histograms, three modular distributions, namely Gamma, inverse Gaussian and lognormal, were fitted to the globule sizes and inverse Gaussian distribution was selected to be the best fit based on Bayesian information criterion. Fig. 9 includes a typical histogram plot of fat globule sizes and the fitted inverse Gaussian distribution. Next, we plotted the estimated two parameters, location $\mu$ and scale $\sigma$, of the fitted distribution for the dataset to assess any clustering of parameters for the same macrovesicualr severity scores. As can be seen in Fig. 10, no significant clustering of the parameters was observed for different scores for our dataset. Next we assessed the area \% contributed by fat globules of different sizes. Fig. 11 shows plots of globule sizes vs. area contributed in percentage for four scores. For scores of 0 and 1 , more fat is contributed by globules of smaller sizes whereas for higher scores of 2 and 3 the percentage of fat area contributed by larger fat globules starts to increase.

\section{Fat Distribution w.r.t. Anatomical Structures}

In this set of experiments, we investigated two spatial properties of fat globules: (a) the uniformity/homogeneity of the spatial dispersion of fat globules, (b) the proportion of fat globules lying within a certain distance to the nearest $\mathrm{CV}$ and PT. Fig. 12 includes plots between the distance to the nearest PT/CV and the percentage of total fat volume lying at that distance. Although, our data set consists of few sample sizes per score value, we make the following observations about the properties of fat globules based on these plots:

1) Fat globules tend to cluster around anatomical structures PT and CV and thus depict inhomogeneous spatial 
distribution.

2) For all severity scores, most of the fat area lies within 1000 microns of PTs and CVs.

3) Fat globules tend to cluster closer to CVs than PTs.

4) For higher severity scores, fat globules tend to spread far from the anatomical structures - thus resulting in heavier tails in these plots with higher scores.

\section{Challenges And Future Work}

In this paper, we presented preliminary results on the detection and spatial analysis of fat globules in 38 digital liver biopsy images. We proposed a dictionary-based, sparse shape recovery algorithm for globule detection specifically to have good results in the presence of challenging biopsy image features such as tissue damage, cell ballooning and clustering globules. Although, in the absence of a benchmark dataset, we do not claim to have better fat detection results as compared to prior work, the validation studies showed promising correlation between the algorithm and other steatosis scoring methods. We indicated that the dictionary-based detection offers the benefit of semi-supervised, online training methods where the algorithm can improve gradually with the minimal contribution by a pathologist to mark and include missed globule instances to the dictionary. In future, the current work can be extended by improving the fat detection including global/neighborhood tissue features as well. This will be particularly useful to reduce false positives due to white spaces having circular shapes but not belonging to fat globules. Since the anatomical markup and pathologist's scores are not always readily available, algorithms can be proposed to detect anatomical markup (CV's and PT's) automatically. Finally, further spatial analysis based on spatial point processes and conducted on bigger dataset can be conducted to investigate any possible spatial patterns specific to scores, patients or outcomes indicating any potential correlations between the pathological symptoms and/or patient histories and disease outcomes. Ultimately, such spatial patterns could have the possibility of being used as biomarkers.

\section{ACKNOWLDGEMENT}

The pathology dataset used for this research was gathered under the ethical permit [M72-07 T5-08] and the research grant [VR/M 2007-2884] from the National Medical Research Council, Sweden, awarded to Peter Lundberg from CMIV, Linköping University. The author would like to thank Claes Lundström and Peter Lundberg from CMIV, Linköping University for useful discussions, Martin Svenson from Sectra, AB, Linköping, for providing with the online biopsy annotation software, Patrik Nasr from Linköping Universitetssjukhuset, Sweden, and Darren Treanor and Tim Palmer from Leeds University, UK, for providing anatomical markup, macrovesicular scores and very useful medical insights into liver pathology. This research was conducted under the VINNOVA grant no. 2014-04257 from the Swedish innovation agency as part of CMIV's digitized pathology project.

\section{REFERENCES}

[1] S. G. Hbscher. Histological assessment of non-alcoholic fatty liver disease. Histopathology, 49(5): 450-465, 2006

[2] Meihong Deng, U. Dahmen, Jian Sun, Hai Huang, C. Sehestedt, A. Homeyer, A. Schenk, and O. Dirsch. Limited correlation between conventional pathologist and automatic computer-assisted quantification of hepatic steatosis due to difference between event-based and surfacebased analysis. IEEE J. of Biomedical and Health Informatics, 18(4): 1473-1477, July 2014.

[3] M. Ishikawa, N. Kobayashi, H. Komagata, K. Shinoda, M. Yamaguchi, T. Abe, A. Hashiguchi, and M. Sakamoto. An accurate method of extracting fat droplets in liver images for quantitative evaluation. Proc. SPIE 9420, Medical Imaging 2015: Digital Pathology, 94200Y, 2015.

[4] G. E. Liquori, G. Calamita, D. Cascella, M. Mastrodonato, P. Portincasa, and D. Ferri. An innovative methodology for the automated morphometric and quantitative estimation of liver steatosis. Gastroenterology, 24(1): 4960, 2009.

[5] N. I. Nativ, A. I. Chen, G. Yarmush, S. D. Henry, J. H. Lefkowitch, K. M. Klein, T. J. Maguire, R. Schloss, J. V. Guarrera, F. Berthiaume, and M. L. Yarmush. Automated image analysis method for detecting and quantifying macrovesicular steatosis in hematoxylin and eosinstained histology images of human livers. Liver Transplantation, 20(2): 228-236, 2014.

[6] A. Zaitoun, M. Al, S. Awad, S. Ukabam, S. Makadisi, and C. Record. Quantitative assessment of fibrosis and steatosis in liver biopsies from patients with chronic hepatitis C. J. of Clinical Pathology, 54(6): 461465, 2001.

[7] T. H. Boyles, S. Johnson, N. Garrahan, A.R. Freedman, and G.T. Williams. A validated method for quantifying macrovesicular hepatic steatosis in chronic hepatitis C. Analytical and Quantitative Cytology and Histology, 29(4): 244-250, 2007.

[8] M. J. Lee, P. Bagci, J. Kong, M. B. Vos, P. Sharma, B. Kalb, J. H. Saltz, D. R. Martin, N. V. Adsay, A. B. Farris. Liver steatosis assessment: Correlations among pathology, radiology, clinical data and automated image analysis software. Pathology - Research and Practice, 209(6):, 371-379, 2013.

[9] X. Ma, N.-S. Holalkere, A. Kambadakone R., M. Mino-Kenudson, P. F. Hahn, and D. V. Sahani. Imaging-based quantification of hepatic fat: Methods and clinical applications. RadioGraphics, 29(5): 1253-1277, 2009.

[10] Jun Kong, M. J. Lee, P. Bagci, P. Sharma, D. Martin, N. V. Adsay, J. H. Saltz, and A. B. Farris. Computer-based image analysis of liver steatosis with large-scale microscopy imagery and correlation with magnetic resonance imaging lipid analysis. In IEEE Intl. Conf. on Bioinformatics and Biomedicine, proceedings, pp. 333-338, Nov. 2011.

[11] G. A. Sigurdsson, Z. Yang, T. D. Tran, J. L. Prince. Interpretable exemplar-based shape classification using constrained sparse linear models. In SPIE Medical Imaging: Image Processing, proceedings, 94130R, 2015.

[12] V. Ratziu, F. Charlotte, A. Heurtier, S. Gombert, P. Giral, E. Bruckert, A. Grimaldi, F. Capron, and T. Poynard. Sampling variability of liver biopsy in nonalcoholic fatty liver disease. Gastroenterology, 128(7): 1898-1906, 2005.

[13] R. B. Merriman, L. D. Ferrell, M. G. Patti, S. R. Weston, M. S. Pabst, B. E. Aouizerat, and N. M. Bass. Correlation of paired liver biopsies in morbidly obese patients with suspected nonalcoholic fatty liver disease. Hepatology, 44(4): 874-880, 2006.

[14] S. Bonekamp, A. Tang, A. Mashhood, T. Wolfson, C. Changchien, M. S. Middleton, L. Clark, A. Gamst, R. Loomba, and C. B. Sirlin. Spatial distribution of MRI-determined hepatic proton density fat fraction in adults with nonalcoholic fatty liver disease. J. of Magnetic Resonance Imaging, 39(6): 1525-1532, 2014.

[15] C. Tomasi and R. Manduchi. Bilateral filtering for gray and color images. In ICCV, proceedings, pp. 839-846, Jan 1998

[16] R. Marcos, R.A. Monteiro and E. Rocha. The use of design-based stereology to evaluate volumes and numbers in the liver: a review with practical guidelines. J. of Anatomy, 220(4): 303-317, 2012.

[17] E. Goceri, Z. K. Shah, R. Layman, X. Jiang, and M. N. Gurcan. Quantification of liver fat. Comput. Biol. Med., 71, C): 174-189, April 2016. 